\title{
First Results on the Fast Neutral Lithium Beam Diagnostics Probing the Edge Plasma of the ETE Tokamak
}

\author{
Rogério Moraes Oliveira, Mário Ueda, and Luiz Ângelo Berni \\ Instituto Nacional de Pesquisas Espaciais - INPE \\ Laboratório Associado de Plasmas, 12227-010, São José dos Campos, SP, Brazil
}

Received on 2 February, 2004; revised version received on 30 April, 2004

\begin{abstract}
One of the main objectives of the research program at ETE is devoted to the study of confinement properties in a low aspect ratio tokamak plasma configuration, and to attain this purpose the determination of plasma parameters of the edge region plays an essential role. A Fast Neutral Lithium Beam (FNLB) diagnostics was developed to perform the measurement of the density profile and its fluctuations at the edge of the plasma in ETE. The first light signals were collected after the filling of ETE chamber with He gas $\left(10^{-4}\right.$ Torr), where the optimization of this diagnostics was done, to adjust the better point for focalization of the beam and for the optical detection system setup. An extensive study of the variation of the intensity of the light signal with the variation of the gas pressure was carried out. This calibration will be used during shots in ETE to measure the rapidly varying pressure of the pulsed discharge by FNLB technique, a requirement imposed by the use of puff valves for injection of the gas. This paper will also describe the several phases of the development of this diagnostics, discuss the problems occurred and the adopted solutions and show the first measurements of the probing of the edge plasma of ETE with FNLB.
\end{abstract}

\section{Introduction}

Plasma boundary study in tokamaks became an important issue recently due to the consensus that many phenomena related to the plasma core are correlated with the conditions of the plasma edge [1-3]. However, this region is characterized by steep gradients of the plasma parameters [4] and by high contamination due to plasma-wall interaction [5], being difficult to be diagnosed. Hence, to conveniently probe the edge plasma, the chosen diagnostics system should fulfill the requirements of allowing high spatial and temporal resolution during measurements, no contamination of the plasma and low disturbance on the discharge. These characteristics, that are inherent to the Fast Neutral Lithium Beam Probes (FNLBP), disseminated the use of this diagnostics to the edge density measurement purposes in some tokamaks and helical systems with various device sizes [6-11] around the world.

A $60 \mathrm{keV}$ mono-energetic beam of neutral $\mathrm{Li}$ atoms was used to probe the top edge region of the JET plasma. There, the radiation emitted from a $17 \mathrm{~cm}$ long section of the beam path is sampled employing an array of 50 optical fibers and fast CCD cameras. Plasma background radiation is separated from the resonance beam emission line by a low resolution spectrometer. Recently, a $30 \mathrm{keV}$ lithium beam probe system was used to measure the edge density and its fluctuation in discharges of the LHD device. For the photon detection from the Li atoms, a thermoelectric cooled CCD camera is used, and a $1 \mathrm{~nm}$ band-pass filter is installed between the camera lens and the CCD chip. In this experiment, time resolution, i.e., exposure time of $\mathrm{CCD}$ is $10-100 \mathrm{~ms}$. Inno- vative two-dimensional neutral lithium beam probe was designed and installed on the CHS device [1]. In this case the injected beam line is mechanically tilted to cover the edge region of the plasma two-dimensionally. Light emission from the $10-20 \mathrm{keV}$ injected beam is collected by an eight-channel detector system using optical band-pass filters and avalanche photodiodes. High energy Li beam (60keV$100 \mathrm{keV}$ ) was used to probe the plasma of the ASDEX tokamak. Interference filter and photomultiplier were used for the collection of light emitted from the lithium resonance line. At TEXTOR, thermal $\mathrm{Li}$ and He beams and fast neutral lithium beam probed the edge plasma region. Both, interference filter - photomultiplier and/or interference filter CCD camera combinations, were used to monitoring the line emission intensity along the injected beam. Separation of $\mathrm{Li}$ beam induced line emission from the plasma background radiation is made via beam modulation.

In the particular case of the ETE tokamak [12] (major radius $\mathrm{R}=0.30 \mathrm{~m}$, aspect ratio $A=1.5$, designed plasma current of 200KA with a toroidal magnetic field up to $0.4 \mathrm{~T}$ ), a FNLB was designed to probe its edge region. The beam is operated at an energy of $15 \mathrm{keV}$ with a neutralization efficiency of $80 \%$. The lithium ion source is a vitreous $\beta$ eucryptite $[13,14]$. The principle of the determination of the desnsity lies in the fact that the intensity of the Li resonance line ( $6708 \AA$ ) emitted from the probing beam due to the interaction with plasma electrons and ions is proportional to the local electron density [15]. The one-channel optical detection system being used is composed of camera lens, optical fiber, interference filter ( $F W H M=100 \AA)$ and photomultiplier tube. 


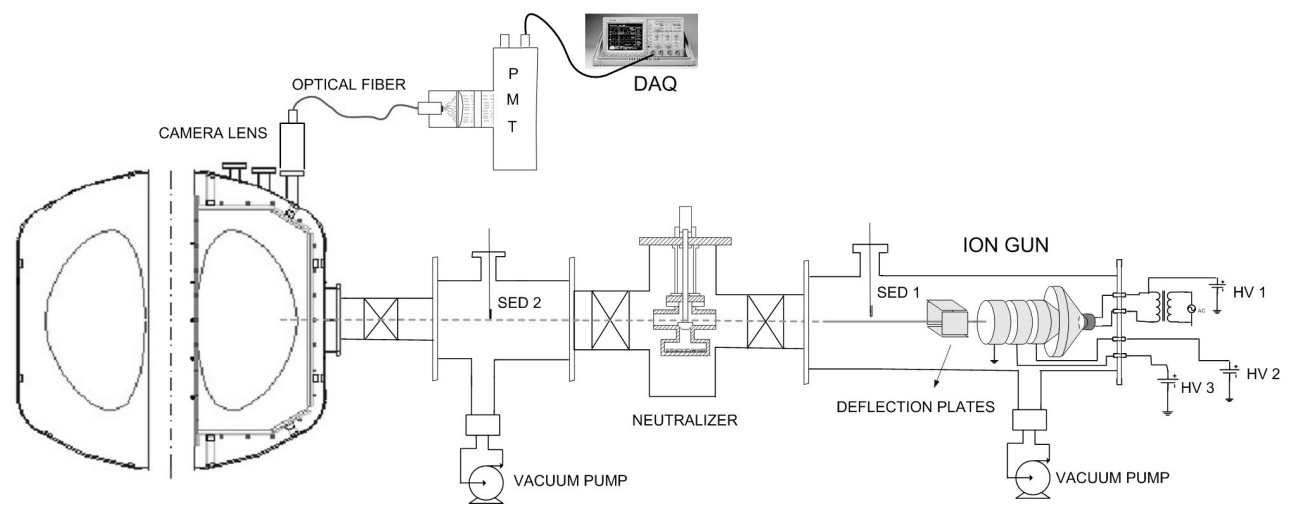

Figure 1. Detailed view of the FNLB probing the edge plasma of the ETE tokamak.

In a comparison to other FNLB devices previously cited, main advantages in favor of the FNLB device installed in ETE are the good spatial resolution, of less than $1 \mathrm{~cm}$, due to the relatively low energy of the acceleration voltage of the injected beam, and excellent time resolution during measurements, of the order of $1 \mu \mathrm{s}$, due to the use of photomultiplier tube. When high energy beams are used, as is the case in JET, ASDEX, LHD, the plasma can be probed more deeply but with the disadvantage of worst spatial resolution. For the case of a $60 \mathrm{keV}$ acceleration voltage for example, spatial resolution is of the order of $3 \mathrm{~cm}$. On the other hand, modulation of the beam itself limits the achievable time resolution, that is not better than $2-3 \mathrm{~ms}$, as it happens at LHD, CHS, TEXTOR. CCD cameras allow excellent spatial resolution in comparison to optical fiber setup (specially fiber bundle), but due to their limited frequency of data acquisition, typically 100 times higher than photomultiplier tubes, time resolution becomes compromised, excluding here their elevate cost.

This paper describes details of the design of the FNLB device installed on ETE tokamak, the calibration process and the first measurements on the edge plasma.

\section{Experimental Setup}

The experimental setup of the FNLB diagnostics may be divided into 3 main parts: the ion gun, the neutralization chamber and the optical detection system.

$\mathrm{Li}$ ions are emitted through the heating of $\beta$ eucryptite compound $\left(\mathrm{Li}_{2} \mathrm{O} \cdot \mathrm{Al}_{2} \mathrm{O}_{3} \cdot 2 \mathrm{SiO}_{2}\right)$ to temperature over $1100^{\circ} \mathrm{C}$ in a self heated molybdenum crucible of $0.6 \mathrm{~mm}$ diameter and $2 \mathrm{~mm}$ depth. This crucible is housed in a Pierce gun, being submitted to a potencial of $15 \mathrm{kV}$. Through the application of adequate potentials to tree electrostatic cylindrical lenses, the beam is accelerated and focused. The 3 high voltage DC power supplies being used for this purpose are connected to the cylindrical lenses through special resistors in order to limit the current delivered in the case of sparks. The heating of the crucible is made via $\mathrm{AC}$ voltage, where a $20 \mathrm{kV}$ insulation transformer is used to insulate the high voltage region from the electric distribution system. Small corrections on the trajectory of the beam may be done by polarizing the deflection plates situated straight in front of the cylindrical lenses, as it can be shown in Fig. 1.

The neutralization system, situated at a distance of $70 \mathrm{~cm}$ from the gun, is composed of a neutralizer cell that accommodates small pieces of metallic sodium, a $12 \mathrm{~cm}$ pass through tube and a pneumatic valve that seals the neutralizer cell. When heated to temperature above $220^{\circ} \mathrm{C}$ the sodium is vaporized, filling the pass through tube when the valve is opened. Through charge exchange, the Li beam is neutralized. In order to avoid the migration of sodium to the ETE chamber and to the ion gun, two collimators with apertures of $2.5 \mathrm{~cm}$ diameter are placed at the entrance tube and at the end of the tube of the neutralizer chamber. The neutralizer housing is also separated from the ion gun and from the tokamak window by two pneumatic gate valves, that are opened for a few seconds just when the discharge is to be initiated, as it occurs also with the pneumatic valve. Differential pumping is guaranteed by two strategically located turbomolecular pumps.

The optical detection system is composed of an adjustable camera lens focused onto the tip of a $7 \mathrm{~m}$ fiber bundle (4 rows of 22 fibers $x 3$ rows of 21 fibers, $\phi=5 \mathrm{~mm}$ ), connected to a plano-convex lens, interference filter $(F W H M=$ $10 \mathrm{~nm}$ ) and to a photomultiplier tube. The output signal, presently acquired in a digital oscilloscope, will be captured by the data acquisition system of ETE [16], based on VME bus.

\section{Beam Characterization}

In order to be possible the characterization of the beam, a set of current detectors were disposed in the FNLB vacuum chamber.

The monitoring of the ion current density was performed with the aid of tree secondary electron detectors (SED ${ }_{1}$, $\mathrm{SED}_{2}$ and $\mathrm{SED}_{3}$ ). These SED's were positioned respectively at the end of the ion gun tube, at the neutralizer region and at the end of the flight tube of the vacuum chamber (near the entrance window of the tokamak), playing distinct roles. The maximum ion current that could be extracted from the gun was monitored by $\mathrm{SED}_{1}$. Through the variation of the potencial applied to the electrostatic lenses and the variation 
in the distance among them, a maximum ion current of $1 \mathrm{~mA}$ was detected by $\mathrm{SED}_{1}$. In this case the variation of the potencial of the electrostatic lens responsible for focusing the beam did not interfere on the current detected by SED $_{1}$ due to its proximity to the ion gun. Otherwise, the effects of divergence of the beam could be monitored in $\mathrm{SED}_{2}$, where the polarization of the focusing lens was indeed significant, as can be seen in the graph of Fig. 2.

In Fig. 2, it is possible to observe that a maximum current of $42 \mu \mathrm{A}$ was detected by $\mathrm{SED}_{2}$ against an ion current detected by $\mathrm{SED}_{1}$ of $240 \mu \mathrm{A}$. This six fold decrease on the current detected is mainly due to the divergence of the beam, caused by space charge effects.

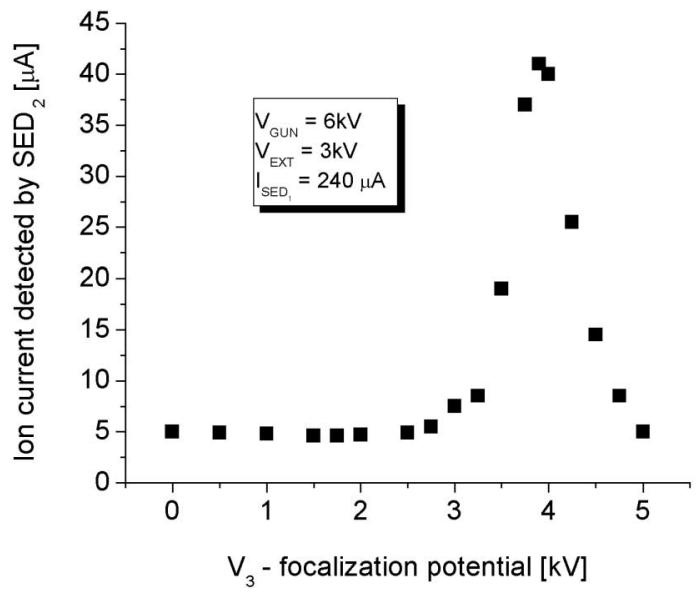

Figure 2. Variation of the ion current detected at the region of the neutralizer against the Focusing potential $\mathrm{V}_{3}$.

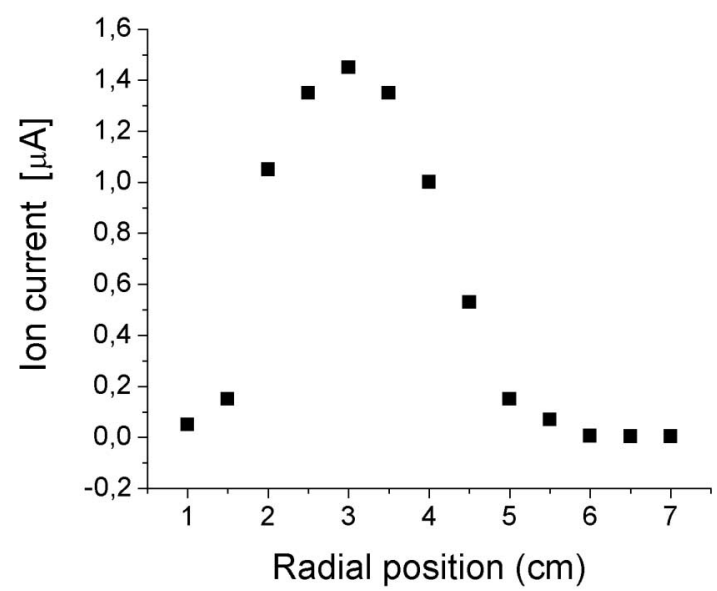

Figure 3. Typical ion beam profile measured at the neutralizer chamber.

At the entrance window of the tokamak, the ion current density detected by $\mathrm{SED}_{3}$ is of $30 \mu \mathrm{A} / \mathrm{cm}^{2}$. This is approximately the value of the ion current density of the beam that will probe the edge plasma in the tokamak.
Other type of current detector, made with a thin cupper rod, was used to measure the beam profile at the neutralizer region, that is strongly dependent on the divergence of the beam. The polarization of the lenses of the ion gun is adjustable in accordance with the beam profile measurement.

A typical measurement of the beam profile at the neutralizer region, in which the FWHM is equal to $2 \mathrm{~cm}$ is shown in Fig. 3.

\section{Neutralization efficiency measure- ment}

One of the most important characteristics of FNLB diagnostics systems is related with the rate of the neutralization of the Li ions, being it strongly dependent on the energy of the beam.

The neutralization efficiency measurement for the $15 \mathrm{KeV}$ lithium beam in ETE was performed in two stages. As it is shown in the experimental scheme of Fig. 4, a strong electric field $(200 \mathrm{~V} / \mathrm{cm})$ produced by the polarization of deflection plates, totally deviates the ion beam to the secondary electron detector 3 (SED 3). When the neutralizer is on, the rate of the ion current detected by SED 3 decreases, accordingly to the increase on the temperature of the neutralizer cell. In fact, the current $I_{D}$ detected by SED 3 is equal to : $I_{D}=I_{b}^{+}+\gamma I_{b}^{+}$. Here $I_{b}^{+}$is the beam ion current and $\gamma$ is the secondary electron emission coefficient. In the same way, the current detected in SED 2, after the neutralization, is equal to: $I_{D}=\gamma I_{b}^{0}$, where $I_{b}^{0}$ is the beam neutral equivalent current. The rate of the currents detected by SED 3 and SED 2 is proportional to the neutralization efficiency. However, the value of $\gamma$ must be known in order to be possible to achieve the neutralization efficiency. The second part of the experiment is devoted to the measurement of the value of $\gamma$ for the beam energy of $15 \mathrm{KeV}$. Now, a Faraday cup (F.C.) situated about $120 \mathrm{~cm}$ from the neutralizer, being composed of an outer cylinder connected to the ground, a collector plate and an inner cylinder serving as a polarized grid, measures the ion current $I_{b}^{+}$and equivalent neutral beam current $I_{b}^{0}$ passing through the $40 \mathrm{~mm}$ diameter entrance aperture. When the neutralizer is off and the grid of the F.C. is biased at a negative voltage sufficient to suppress secondary electrons, the detected current $I_{D}$ is equal to $I_{b}^{+}$. On the other hand, when the grid is biased by a positive voltage, the detector registers the current of secondary electrons released from the collector of $\gamma I_{b}^{+}$as well as the ion current $I_{b}^{+}$.

The value of $\gamma=2$ was determined by comparing the beam ion current detected by the F.C. for both positive and negative polarization of the grid.

In Fig. 5 it is possible to observe the variation of the neutralization efficiency with the heating temperature of the neutralizer cell. It can be seen that a maximum of $80 \%$ of neutralization efficiency was obtained for sodium temperature of about $300^{\circ} \mathrm{C}$. The decay of the neutralization efficiency for temperature above this value is due to the excess of sodium vapor produced, that influence the ion emission. 


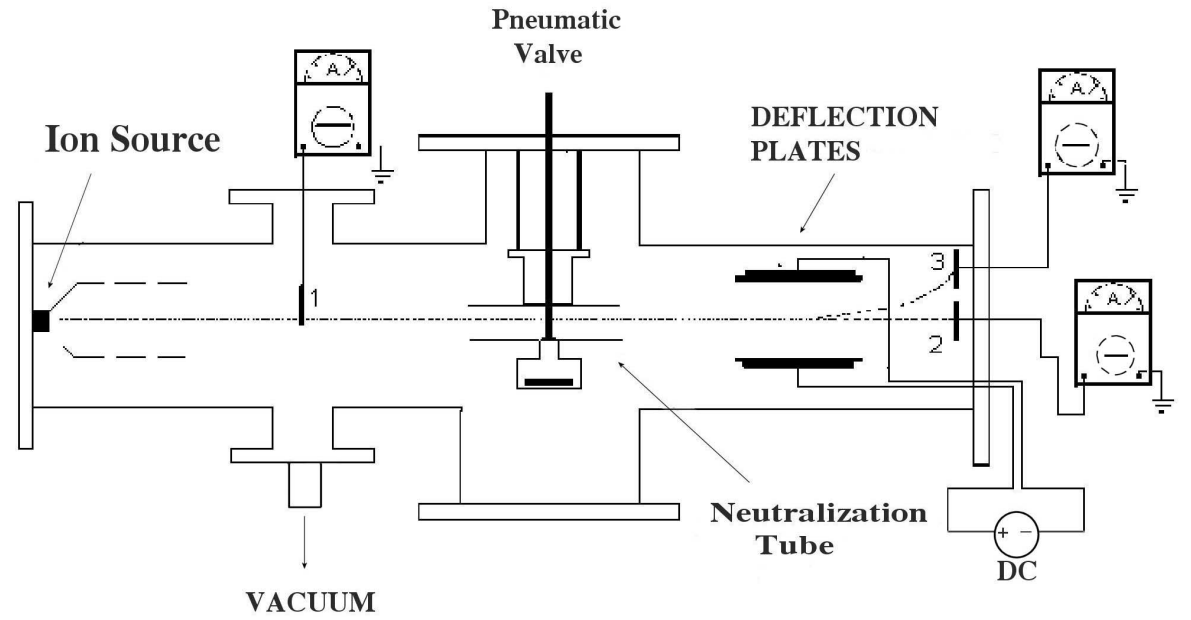

Figure 4. Experimental apparatus used to measure the neutralization efficiency.

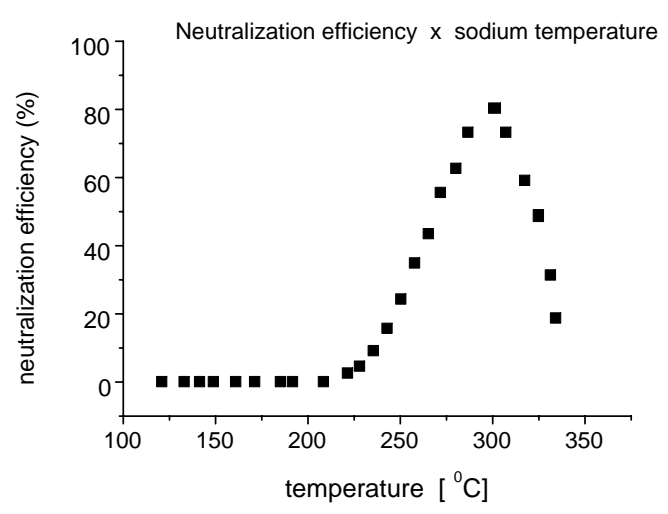

Figure 5. Neutralization efficiency versus sodium temperature for ion beam of $15 \mathrm{keV}$.

\section{The influence of stray magnetic fi- eld}

In the routine operation of ETE a stray magnetic field in the poloidal and toroidal directions of about 10Gauss and 4 Gauss were measured in the region of the neutralizer, respectively.

The resultant ion Larmor radius is equal to $120 \mathrm{~m}$ for the magnetic field of 10 Gauss, according to equation 1 .

$$
r_{L}=\frac{m v_{\perp}}{q B}=\frac{1.15 \times 10^{-26} \times 1.66 \times 10^{6}}{1.6 \times 10^{-19} \times 10 \times 10^{-4}} \approx 120 \mathrm{~m}
$$

It implies a horizontal deviation of $6^{\circ}$ on the trajectory of the beam until it reaches the optical detection window. Similar calculation leads to an ion Larmor radius of $300 \mathrm{~m}$ for the spurious toroidal magnetic field case, that causes the deviation of the beam in the vertical direction of $2.4^{\circ}$. These deviations were observed by the change on the intensity of the light detected by the photomultiplier output signal, as can be seen in Figs. 6 and 7 below.

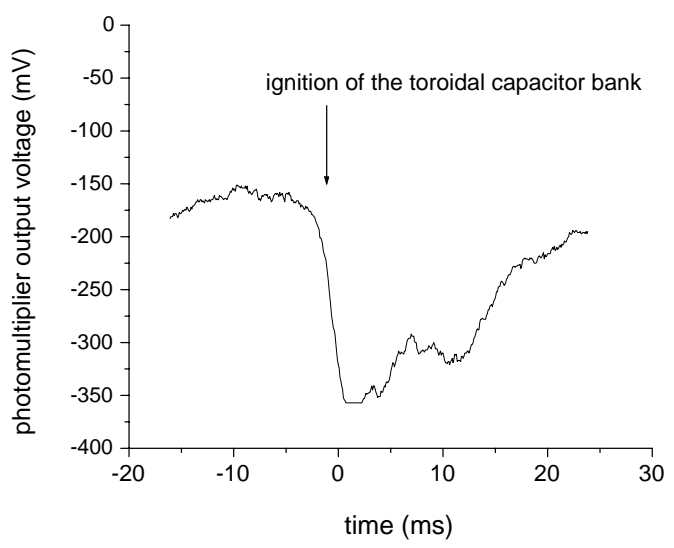

Figure 6. Variation on the amount of light detected by the photomultiplier tube after the formation of the toroidal magnetic field.

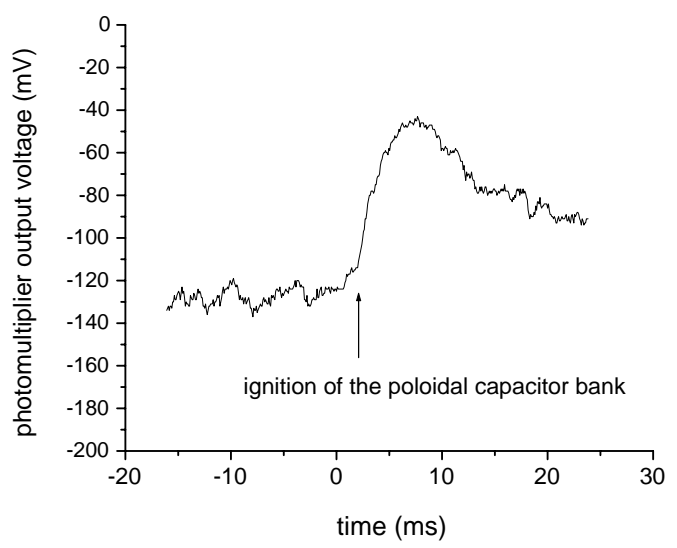

Figure 7. Variation on the amount of light detected by the photomultiplier tube after the formation of the poloidal magnetic field. 
The increase on the intensity of the photomultiplier output signal observed in Fig. 6 is due to the upward deflection of the beam. In this case the light emitted from the beam is shifted to a position closer to the optical window, causing an increase on the signal detected. On the other hand the horizontal deflection of the beam due to the influence of the spurious poloidal field removes partially the emitted light from the observation cone of the optical detection system, causing the reduction of the intensity of the signal, as can be seen in Fig. 7.

Independent tests were made with cylinders of similar diameters to the ones used on the FNLB chamber, with permanent magnet in order to produce magnetic field varying from 10 to 100Gauss. An external shielding consisting of a $1 \mathrm{~mm}$ thick cylinder of $\mu$-metal caused a fourfold decrease to the measured field in the shielded region.

Based on this test, the ion source chamber, the flight tubes and the neutralizer chamber were involved by a $1 \mathrm{~mm}$ thick cylinder of $\mu$-metal. This led to a reduced vertical and horizontal deflections on the beam trajectory to $1.5^{\circ}$ and $1^{\circ}$, respectively, with reduction of the variation of the output signal of the photomultiplier. These deviations are small enough not to interfere on the measurement process performed by this diagnostics.

\section{Calibration process}

First light signals were collected after the filling of ETE chamber with He gas and consequent interaction of the lithium atoms with neutral particles. A sweep around the lithium resonance line ( $6708 \AA$ ) was performed in order to verify the distribution of the intensities of wavelengths detected by the optical detection system. In Fig. 8 it is possible to observe that the wavelength distribution is centered at $6708 \AA$, in which wavelength the maximum intensity of the signal occurs, as it was expected.

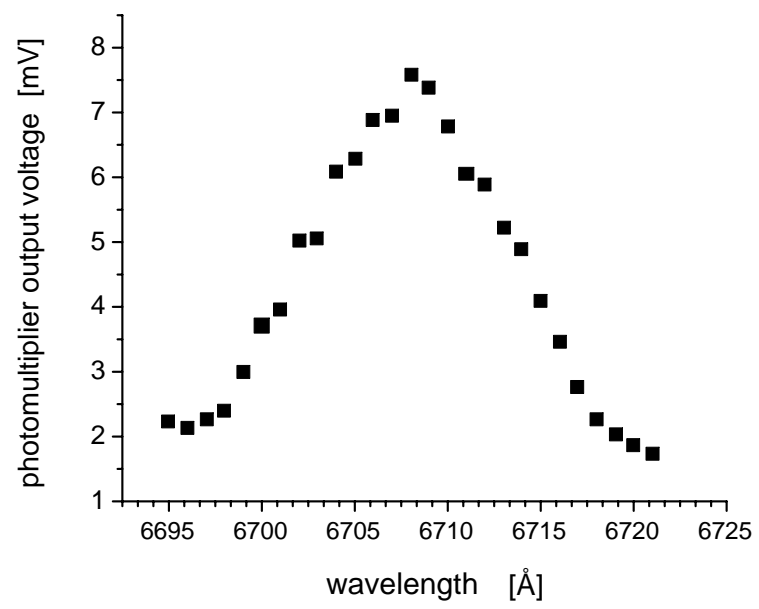

Figure 8 - Sweep around $6708 \AA$ resonance line for Li beam injected in He gas at $4 \times 10^{-4}$ Torr.

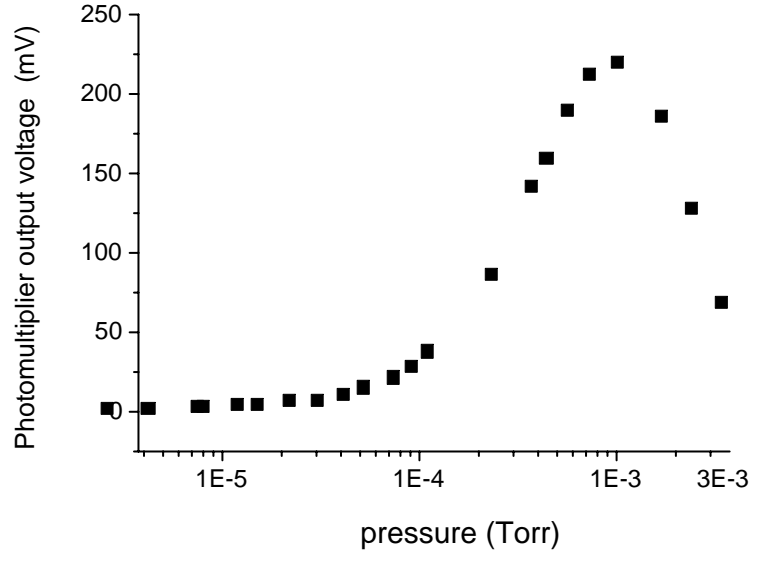

Figure 9. Intensity of the Li resonance line for injection of the beam in hydrogen gas at different pressures.

The second step performed in the calibration process was done by varying the pressure inside the chamber and verifying the variation of the intensity of the output signal of the photomultiplier. The range of variation of the intensity of the light signal collected in response to the variation of the gas pressure begins at $4 \times 10^{-6}$ Torr, reaching pressures as high as $3 \times 10^{-3}$ Torr. As may be seen in Fig. 9, the maximum intensity of the signal occurred for $p=1 \times 10^{-3}$ Torr. The excess of collisions of lithium atoms with neutral particles existing for higher values of the pressure causes the attenuation of the beam, with consequent reduction of the intensity of the emission of light. Notice that pressure of operation in ETE is in the range of $10^{-5}$ to $10^{-4}$ Torr. This fact promotes the FNLB as an excellent pressure meter for ETE discharges, since it makes it possible to measure the fast varying pressure in ETE chamber when puff valves are used for the injection of gas. In fact, several measurements with FNLB were performed by injecting gas through puff valves into ETE chamber. A typical signal showing the exact instant of the fast input of gas into the chamber and the consequent reduction of the pressure due to the vacuum pumping may be seen in Fig. 10.

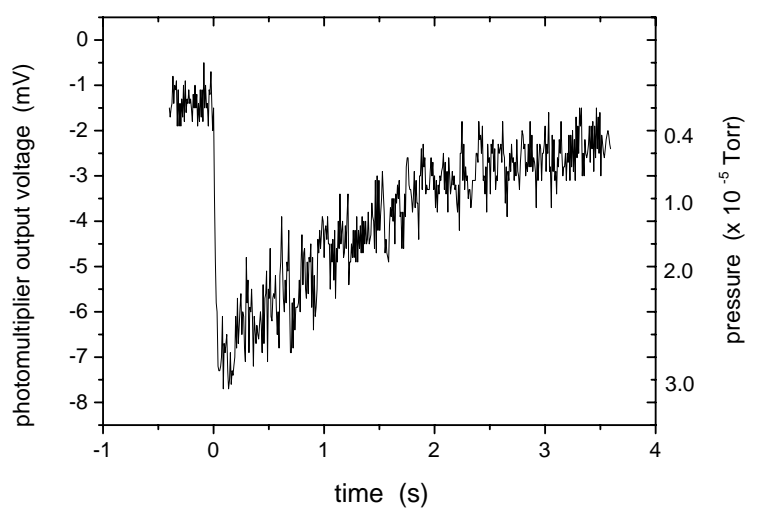

Figure 10. Measurement of the varying pressure in ETE chamber during the injection of the gas by puff valve and evacuation due to vacuum pumping. 


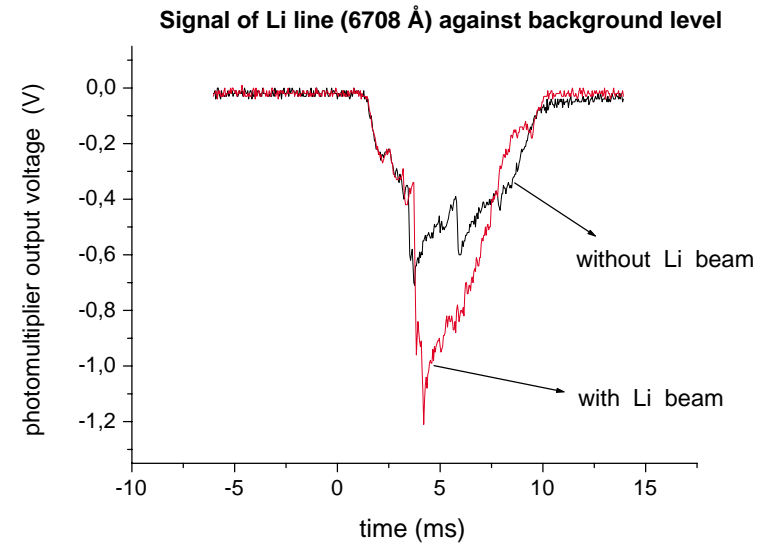

Figure 11. Intensity of Li resonance line radiation against background level.

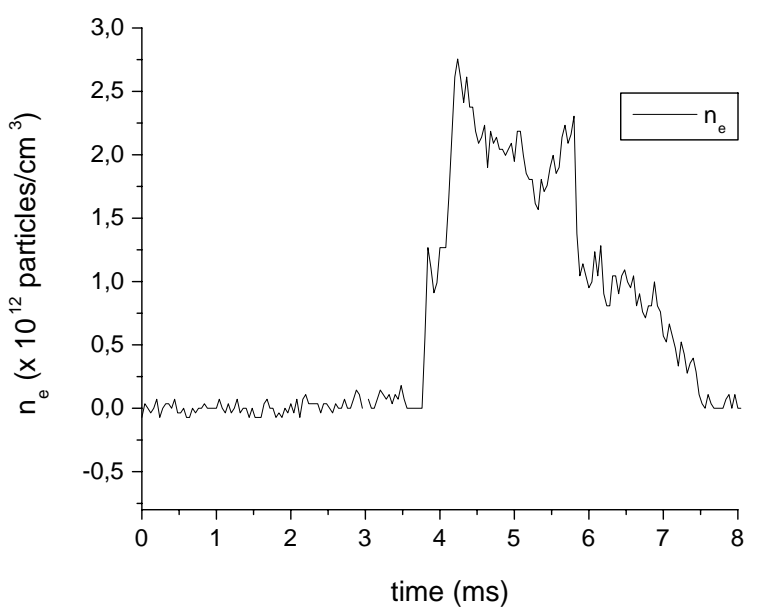

Figure 12. Temporal evolution of the plasma density for $R=48 \mathrm{~cm}$

\section{Measurements at the plasma}

The intense background radiation emitted from the plasma, mainly due to $\mathrm{H} \alpha$ emission, is just partially cut off by the optical detection system, being this noise level several times higher than the emitted line radiation $(6708 \AA)$ of interest. In order, to measure the correct plasma density we must perform two sequential shots, while maintaining plasma conditions. The first one is normally made without the application of the beam, just to allow the measurement of the noise level. The difference on the intensity of both signals (with and without the application of the beam) is proportional to the electron density of the plasma under the following conditions: plasma temperature $T_{e}<100 \mathrm{eV}$ and low attenuation of the Li beam. These conditions have been observed at the outer window position $(R=48 \mathrm{~cm})$ and for the present status of development of ETE [17] (the energy delivered by the capacitor banks represents approximately $\frac{1}{4}$ of the total energy expected for the initial stage of operation of ETE). In this radial position, the temperature measured by Thomson Scattering diagnostics remained always below $30 \mathrm{eV}$ [18]. The principle of density determination used for this situation was based on the comparison of the photon flux profiles emitted from the lithium atoms due to interaction with plasma electrons $N_{v p}$ and due to the interaction with hydrogen gas $N_{v g}$ under same discharge conditions [19]. Here, the electron density is deduced from the relation described in equation 2 [6].

$$
n_{e}=\frac{N_{\nu p}}{N_{\nu g}} \frac{Q_{g}}{Q_{p}} n_{g}
$$

where: $Q_{g}$ is the cross section for interaction of lithium atoms with molecular gas, $Q_{p}$ is the effective cross section for collision of lithium atoms with the electrons of the plasma and $n_{g}$ is the number density of the gas.

In the first instance the density of a glow discharge using He gas at $2 \times 10^{-4}$ Torr was measured. In this case the continuous intensity of the signal collected when lithium beam was injected into the discharge was $90 \%$ higher than the signal measured without the injection of the beam. From known cross section data [15] and calculation of the gas number density $n_{g}$, the obtained plasma density was of $2 \times 10^{11}$ particles $/ \mathrm{cm}^{3}$.

The time evolution of the plasma density for typical ETE discharges was also obtained for $R=48 \mathrm{~cm}$, using the same method described above. The graph of Fig. 11 shows the intensity of the signal collected by the photomultiplier when the beam is injected into the plasma against the background level. The resultant density profile is shown in Fig. 12.

\section{Conclusion}

Several phases of the development of the FNLB diagnostics were overcame. First light signals were collected after the filling of ETE chamber with He gas at $10^{-4}$ Torr, being possible the optimization of the diagnostics and the adjustment of the optical detection system setup. The measurement of the fast varying pressure during injection of gas in ETE chamber by puff valves was obtained. A $15 \mathrm{keV}$ lithium beam of $2 \mathrm{~cm}$ diameter with equivalent current of $30 \mathrm{~mA} / \mathrm{cm}^{2}, 80 \%$ neutralized, is being used to probe the edge plasma of the ETE tokamak. Even though we realize that some important modifications must be done to improve the measurements, the first results showed to be very satisfactory. The main modification to be implemented is the increase of the channels for optical detection, which will allow the measurement of the density for other radial positions. The interference filter being used of $10 \mathrm{~nm}$ pass-band will also be substituted by a $3 \mathrm{~nm}$ pass-band one. This will allow a higher signal to noise ratio during measurements.

In summary, a neutral lithium beam probe system for measurement of the time evolution of the plasma density in ETE has been developed. The measurement of the temporal evolution of the density of the plasma edge with FNLB, of the order of $10^{12} \mathrm{~cm}^{-3}$, must be confronted with data from Langmuir probe for the region near the limiter and with data 
from Thomson Scattering for the inner region. The preparations for this experiment is underway.

\section{References}

[1] K. Nakamura, H. Iguchi, M. Ueda, T. Morisaki, M. Isobe, K. Ida, C. Takahashi, S. Okamura, and K. Matsuoka, Journal of Nuclear Materials, 725 (2003).

[2] M. Ueda et al., Journal of Nuclear Materials, 923 (1992).

[3] P. Varela and M. E. Manso, Rev. Sci. Instrum. 74, 1493 (2003).

[4] W. M. Stacey, Fusion Technology 36, 38 (1999).

[5] E. Hintz, Transactions of Fusion Technology 25, 333 (1994).

[6] H. Iguchi, K. Kadota, K. Takasugi, T. Shoji, M. Hosokawa, M. Fujiwara, and H. Ikegami, Rev. Sci. Instrum. 56, 1050 (1985).

[7] A. Komori, O. Mitarai, K. Yamagiwa, C. Honda, K. Kadota, and J. Fujita, Nucl. Fusion 28, 1460 (1988).

[8] D. M. Thomas, Rev. Sci. Instrum. 66, 806 (1995).

[9] T. Morisaki, Plasma Phys. Controlled Fusion 37, 787 (1995).

[10] T. Morisak, A. Komori, O. Motojima and LHD Experimental Group, Rev. Sci. Instrum. 74, 1865 (2003).
[11] K. McCormick, M. Kick, "The ASDEX $100 \mathrm{keV}$ Neutral Lithium Beam Diagnostic Gun”, IPP Report IP III/85, MaxPlanck Institute, Garching (1983).

[12] G. O. Ludwig, E. Del Bosco, J.G. Ferreira, L.A. Berni, R.M. Oliveira, M.C.R. Andrade, C.S. Shibata, M. Ueda, L.F.W. Barbosa, J.J. Barroso, P.J. Castro, and H. Patire Jr, Braz. J. Phys. 33, 848 (2003).

[13] M. Ueda, R. R. Silva, R. M. Oliveira, H. Iguchi, J. Fujita, and K. Kadota, J. Phys. D: Appl. Phys. 30, 2711 (1997).

[14] R. M. Oliveira, J. A. N. Gonçalves, M. Ueda, and A. F. Beloto, Japanese Journal of Applied Physics 43, 1154 (2004).

[15] K. Kadota, K. Tsuchida, Y. Kawasumi, and J. Fujita, Plasma Physics 20, 1011 (1978).

[16] R. M. Oliveira, L. F. W. Barbosa, J. G. Ferreira, and C. S. Shibata, Fusion Engineering and Design 60, 403 (2002).

[17] L. A. Berni, E. Del Bosco, R. M. Oliveira, and M. P. Alonso, Braz. J. Phys. 34, 1572 (2004).

[18] L. A. Berni, M. Ueda, E. Del Bosco, J. G. Ferreira, R. M. Oliveira, and W. A. Vilela, Rev. Sci. Instrum. 74, 1200 (2003).

[19] R. M. Oliveira, M. Ueda, and W. A. Vilela, Braz. J. Phys. 32, 76 (2002) 